\title{
Hand dermatitis with Hanseniaspora uvarum as a plausible causative agent
}

\author{
Marek Jankowski ${ }^{1}$, Tomasz Jagielski ${ }^{2}$, Grażyna Misiak ${ }^{3}$, Rafał Czajkowski ${ }^{1}$
}

${ }^{1}$ Chair of Dermatology, Sexually Transmitted Diseases and Immunodermatology, Faculty of Medicine in Bydgoszcz, Nicolaus Copernicus University in Torun, Poland

${ }^{2}$ Department of Applied Microbiology, Institute of Microbiology, Faculty of Biology, University of Warsaw, Warsaw, Poland ${ }^{3}$ Department of Microbiological Diagnostics, Provincial Polyclinic Hospital, Torun, Poland

Adv Dermatol Allergol 2018; XXXV (6): 641-643 DOI: https://doi.org/10.5114/ada.2018.72854

The ascomycetes genus Hanseniaspora was created with the description of Hanseniaspora valbyensis in 1912. Yet, the first species from this genus was described as early as in 1870 under the name of Saccharomyces apiculatus [1]. Over the years, a total of six species of Hanseniaspora yeasts have been described. Molecular taxonomy studies have demonstrated Hanseniaspora as the teleomorphic genus of Kloeckera species. Hanseniaspora uvarum is a synonym and valid name of the aforesaid species S. apiculatus, whereas its anamorph is known as Kloeckera apiculata [2].

Hanseniaspora uvarum is commonly found on fresh fruit, and grapes in particular, being an important part of the natural microbiome involved in alcoholic fermentation. The fungus has also been isolated from soil, plants, fruit-eating insects, birds and seafood [3]. The occurrence of $\mathrm{H}$. uvarum in humans is very unusual with only seven documented isolations in the literature [4-7].

Here we present a patient in whom $\mathrm{H}$. uvarum was isolated from the skin lesion, which resolved after antifungal therapy. To the best of our knowledge, this is the first case where $H$. uvarum could be linked to the human pathology.

A 51-year-old, otherwise healthy and immunocompetent Polish woman presented to the dermatologist with a 3-month history of pruritic lesions localized on the dorsal and lateral aspects of the distal interphalangeal joints of the middle and ring finger of her left hand (Figure $1 \mathrm{~A}$ ). The lesions, consisting of small vesicles and occasionally pustules on a relatively well-demarcated erythematous base with lichenification, were inconspicuous and allergic contact dermatitis was suspected. However, neither potential allergen nor irritant agent could be identified from anamnesis, and patch testing with European Baseline Series (Chemotechnique Diagnostics) produced negative results. Empirical treatment with topical 0.1\% mometasone furoate (Elocom) for 2 weeks as well as topical $0.05 \%$ betamethasone dipropionate combined with $0.1 \%$ gentamicin (Bedicort $\mathrm{G}$ ) for 2 weeks resulted in only temporal improvement.

Lesional skin scrapings were collected for bacterial and fungal culture. On MacConkey and Columbia agar with 5\% sheep blood (Oxoid) no bacterial growth was observed. Whereas, on Sabouraud dextrose agar (SDA) with chloramphenicol and gentamicin as well as on SDA with chloramphenicol and cycloheximide (Graso), after 48 -hour incubation at $30^{\circ} \mathrm{C}$, smooth, creamy white, yeast-like colonies were detected (Figure $1 \mathrm{~B}$ ) whose Gram-stained preparation and scanning electron microscopy morphology was merely suggestive of yeast species, showing apiculate, spherical or ovoid cells, with terminal budding (Figures 1 C, D). Identification with the RapID Yeast Plus system (Innovative Diagnostic Systems, USA) produced inconclusive results. The first sample led to the identification of Prototheca zopfii, while the second one, taken from the same colony of the organism, resulted in the identification of either Saccharomyces cerevisiae, Candida intermedia or Geotrichum sp. With the Vitek 2 identification system (BioMérieux, France), the fungus was identified as Kloeckera apiculata (anamorph of Hanseniaspora uvarum). Unequivocal species identification was determined by PCR-sequencing of the rRNA operon loci. This involved extraction of fungal DNA, PCR amplification of the internal transcribed spacer (ITS) regions ITS1 and 2 (i) and the D1/D2 variable domains of the $28 \mathrm{~S}$ rRNA gene (ii), and subsequent sequencing of those loci in both forward and reverse directions, essentially as described elsewhere [8]. The obtained sequences were aligned against the GenBank database using the BLAST algorithm (www.ncbi.nlm.nih.gov/blast/Blast.cgi). The cut-off for species delineation was set at $99 \%$ sequence similarity. The 771-bp ITS1/2 sequence (GenBank acces-

Address for correspondence: Tomasz Jagielski PhD, Department of Applied Microbiology, Institute of Microbiology, Faculty of Biology, University of Warsaw, 1 Miecznikowa St, 02-096 Warsaw, Poland, phone: +48 225541 431, e-mail: t.jagielski@biol.uw.edu.pl Received: 21.08.2017, accepted: 29.08.2017. 

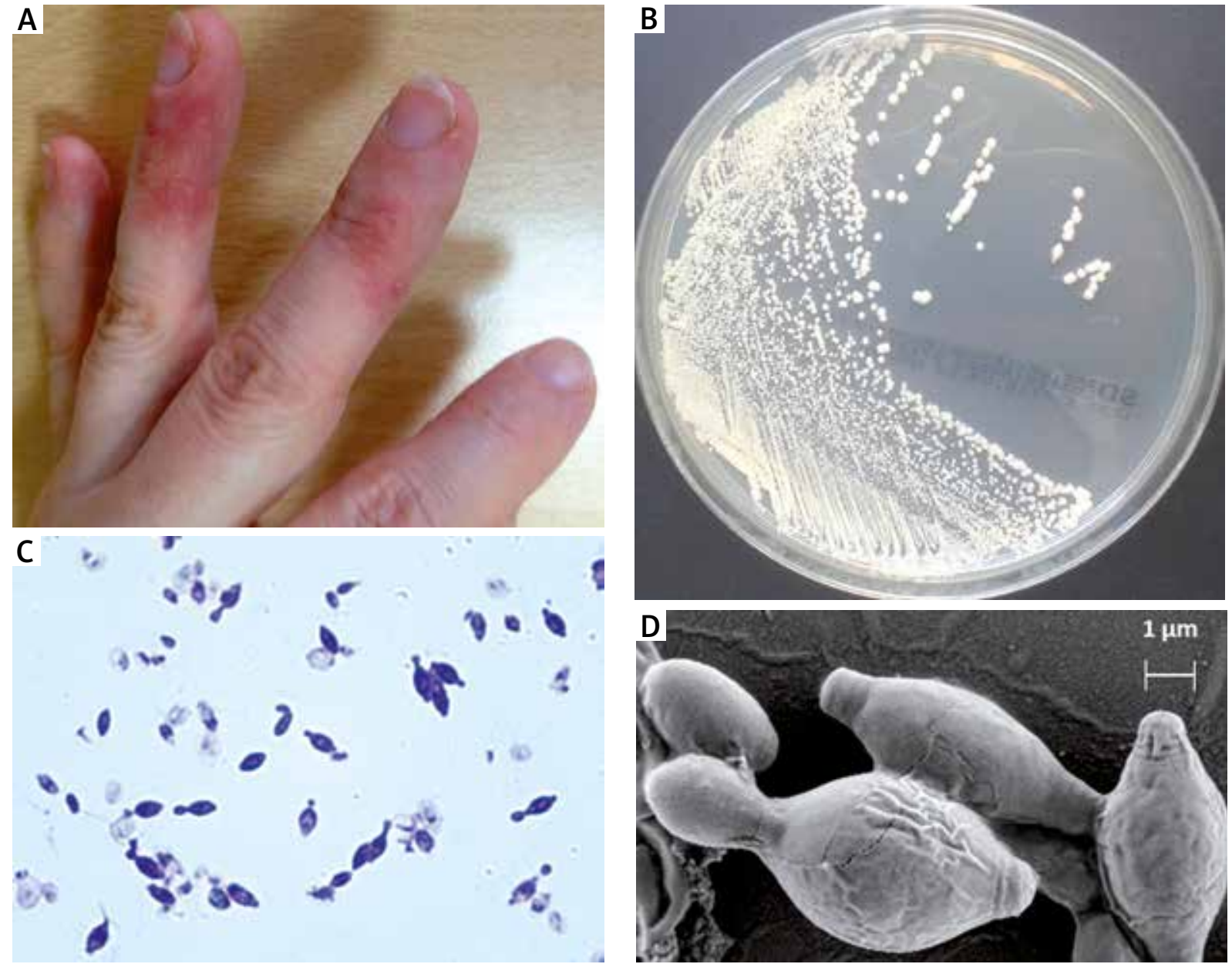

Figure 1. A - Clinical lesions on the middle and ring finger of the patient's left hand. B - Colony morphology after 48-hour incubation on SDA with chloramphenicol and cycloheximide at $30^{\circ} \mathrm{C} . \mathrm{C}-\mathrm{Gram}$ stain preparation from culture; magnification, 100x. D - The same culture upon SEM imaging; magnification 10,000×

sion no. KX400754.1) and the 615-bp D1/D2 sequence (KX400755.1) showed, respectively, 99\% and 100\% identity with the corresponding sequences of Hanseniaspora uvarum available in the GenBank repository.

With the final diagnosis established, the patient was administered $100 \mathrm{mg}$ daily of itraconazole (ITZ) for 28 days, and the lesions resolved without recurrence.

Drug susceptibility testing was performed with the E-test methodology (Liofilchem, Italy), according to the manufacturer's instructions and following the Clinical and Laboratory Standards Institute (CLSI) document M27-S3 [9]. Five antifungal agents were used, namely itraconazole (ITZ), fluconazole (FCZ), voriconazole (VOZ), amphotericin $B(A M B)$, and micafungin (MCF).

Quality control was assured by testing Candida krusei ATCC 6258 and Candida parapsilosis ATCC 22019 reference strains, as recommended by the Clinical and Laboratory Standards Institute (CLSI). The following MIC values were obtained: FCZ, $1.5 \mathrm{mg} / \mathrm{l}$, ITZ, $0.75 \mathrm{mg} / \mathrm{l}$, VOZ,
$0.016 \mathrm{mg} / \mathrm{l}, \mathrm{AMB}, 0.008 \mathrm{mg} / \mathrm{l}$, and MCF, $0.006 \mathrm{mg} / \mathrm{l}$. Given a good clinical response, the drug chosen (ITZ) was not changed when the antibiogram became available.

Recovery of $\mathrm{H}$. uvarum from human tissues is exceptionally rare. So far, it has been documented in four papers only, reporting altogether on seven isolations of H. uvarum from human sources, including nails, feces, oral mucosa, vaginal secretion, and blood [4-7]. The role of $\mathrm{H}$. uvarum in all these cases remains enigmatic. In three of the aforesaid studies, no clinical data were provided, thus precluding any interpretation of finding H. uvarum in human samples. In a study of García-Martos et al. [5], the isolation of the fungus from stool and two ungual specimens was recognized as a transient colonization of the intestine and nails, respectively.

The present case is, to our knowledge, the first one in which human disease can be etiologically linked to H. uvarum. Four observations speak in favor of this assumption. First, the yeast was isolated directly from a le- 
sion. Second, no other pathogenic flora was evidenced. Third, no abnormalities were found upon clinical examination and laboratory testing, which could challenge the infectious nature of the disorder and support any alternative diagnosis. Fourth, the resolution of the lesion was achieved after treatment with itraconazole, a drug commonly used for the treatment of fungal infections, including those of yeast etiology.

No accepted breakpoints for Hanseniaspora spp. are available. Those for Candida spp. reported by CLSI [9] and EUCAST [10] differ significantly. For example, the resistance breakpoints for ITZ were defined as $\geq 1 \mathrm{mg} / \mathrm{l}$ and 0.064 (or 0.125 ) mg/l, respectively. Given the close relationship of Hanseniaspora spp. to Candida spp. [11, 12], the MIC value for ITZ obtained in this study $(0.75 \mathrm{mg} / \mathrm{l})$ might indicate resistance. It is our opinion, however, that such a simple extrapolation of interpretative criteria from one species to another, even closely related, is unjustified and inappropriate, especially in the absence of drug susceptibility studies for Hanseniaspora spp.

Apart from H. uvarum, at least two other Hanseniaspora species have been isolated, yet sporadically, from human samples, that is $H$. guilliermondii [13] and $H$. opuntiae [14]. Through an extensive search of the literature, we found only one case of infection due to Hanseniaspora (valbyensis) fungi. Interestingly, a patient in that case also suffered from hand dermatitis [15]. Of further note is that $H$. uvarum and $H$. valbyensis have almost identical antigenicity as shown by cross agglutination and absorption tests [16].

The emergence of rare fungal pathogens, often displaying complex drug resistance patterns necessitates a precise identification at the species level. Fast and reliable diagnosis is essential for choosing an appropriate and effective treatment. Conventional, phenotype-based methods may leave many yeast fungi unrecognized or misidentified, compared to molecular, DNA-based methods. For rare yeast pathogens, the concordance between these two types of modalities can be as low as $25 \%$ [6]. Also in this report, species identification with commercial kits, designed for biochemical profiling, was inconclusive and required further speciation by means of PCR-sequencing technology. The rDNA-targeted sequencing is a fast and robust procedure, which should always complement the identification path for clinically relevant yeasts, while its use should even be prioritized when those less frequent species are suspected.

To conclude, the case reported herein is the first to prove $\mathrm{H}$. uvarum as the causative agent of human pathology. It is also the first case of Hanseniaspora infection, confirmed by both conventional and molecular approaches.

\section{Conflict of interest}

The authors declare no conflict of interest.

\section{References}

1. Reess M. Botanische Untersuchungen über die Alkoholgährungspilze. A. Leipzig, Felix 1870.

2. Kurtzman CP, Fell JW, Boekhout T. The Yeasts, a Taxonomic Study. Vol. 1. $5^{\text {th }}$ ed. Elsevier, Amsterdam 2011.

3. Albertin W, Setati ME, Miot-Sertier C, et al. Hanseniaspora uvarum from winemaking environments show spatial and temporal genetic clustering. Front Microbiol 2016; 6: 1569.

4. Emmanouil-Nikoloussi E, Kanellaki-Kyparissi M, Papavassiliou P, et al. "Hanseniaspora uvarum" the ultrastructural morphology of a rare ascomycete, isolated from oral thrush. Bull. Group Int Rech Sci Stomatol Odontol 1994; 37: 13-7.

5. García-Martos P, Hernández-Molina JM, Galán F, et al. Isolation of Hanseniaspora uvarum (Kloeckera apiculata) in humans. Mycopathologia 1999; 144: 73-5.

6. Cendejas-Bueno E, Gomez-Lopez A, Mellado E, et al. Identification of pathogenic rare yeast species in clinical samples: comparison between phenotypical and molecular methods. J Clin Microbiol 2010; 48: 1895-9.

7. Severo Gomes B, Souza Motta CM, Lima AN, Porto ALF. Pathogenic characteristics of yeasts isolated from vaginal secretion preserved under mineral oil. J Venom Anim Toxins incl Trop Dis 2011; 17: 460-6.

8. Jagielski T, Rup E, Ziółkowska A, et al. Distribution of Malassezia species on the skin of patients with atopic dermatitis, psoriasis, and healthy volunteers assessed by conventional and molecular identification methods. BMC Dermatol 2014; 14: e3.

9. CLSI. Reference method for broth dilution antifungal susceptibility testing of yeasts; $3^{\text {rd }}$ ed CLSI document M27-A3. Clinical and Laboratory Standards Institute, Wayne, PA 2008.

10. EUCAST Antifungal Agents, Breakpoint tables for interpretation of MICs v. 8.1 valid from 2017-03-01. European Committee on Antimicrobial Susceptibility Testing.

11. Schmalreck AF, Lackner M, Becker K, et al. Phylogenetic relationships matter: antifungal susceptibility among clinically relevant yeasts. Antimicrob Agents Chemother 2014; 58: 1575-85.

12. Pramateftaki PV, Kouvelis VN, Lanaridis P, Typas MA. The mitochondrial genome of the wine yeast Hanseniaspora uvarum: a unique genome organization among yeast/fungal counterparts. FEMS Yeast Res 2006; 6: 77-90.

13. Cadez N, Raspor P, de Cock AW, Boekhout T, Smith MT. Molecular identification and genetic diversity within species of the genera Hanseniaspora and Kloeckera. FEMS Yeast Res 2002; 1: 279-89.

14. Eddouzi J, Lohberger A, Vogne C, et al. Identification and antifungal susceptibility of a large collection of yeast strains isolated in Tunisian hospitals. Med Mycol 2013; 51: 737-46.

15. Batista AC, Coelho RP, Vieira JR. The incidence of Hanseniaspora valbyensis Kloecker in the human cecal appendix and epidermal lesions. Mycopathologia 1960; 12: 185-95.

16. Aksoycan N. The antigenic relationships between Hanseniaspora osmophila, $H$. uvarum and $H$. valbyensis strains. Mikrobiyol Bul 1982; 16: 107-10. 